\title{
Adding Constraint Tables to the DMN Standard: Preliminary Results *
}

\author{
Marjolein Deryck $^{\star \star}$, Bram Aerts ${ }^{\star \star}$, and Joost Vennekens \\ KU Leuven, Department of Computer Science \\ Campus De Nayer, Sint-Katelijne-Waver, Belgium \\ \{marjolein.deryck; b.aerts; joost.vennekens\}@kuleuven.be
}

\begin{abstract}
The DMN standard allows users to build declarative models of their decision knowledge. The standard aims at being simple enough to allow business users to construct these models themselves, without help from IT staff. To this end, it combines simple decision tables with a clear visual notation. However, for real-life applications, DMN sometimes proves too restrictive. In this paper, we develop an extension to DMN's decision table notation, which allows more knowledge to be expressed, while retaining the simplicity of DMN. We demonstrate our new notation on a real-life case study on product design.
\end{abstract}

Keywords. Decision Model and Notation, First Order Logic, Constraint modelling

\section{Introduction}

Recently, the Object Management Group (OMG) has developed a new standard, the Decision Model and Notation [1], as a declarative representation for decision knowledge. It states explicitly that [t]he primary goal of DMN is to provide a common notation that is readily understandable by all business users[...] [1, p.13].

In a recent project $[2$, we used DMN to model the decision process followed by product engineers to design a specific kind of industrial component to match customer requirements. Here, DMN's ability to be understood by "business users" (in this case, the product engineers) was a key advantage. The multinational company with which we collaborate did not have a standardized product design process. Therefore, a significant knowledge elicitation effort was required, in which engineers from all over the world were brought together in order to define a single design process, that could then be partially automated into a decision support system. In these knowledge elicitation workshops, it was key to make use of a formal notation that could be understood by both our knowledge experts and the company's domain experts. This helped to avoid misunderstandings, ensured smooth communication, and allowed certain well-delineated parts

\footnotetext{
* This work is supported by the Flemish Agency for Innovation and Entrepreneurship, TETRA HBC.2017.0039 and R\&D project HBC.2017.0417.

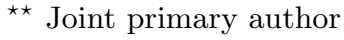


of the decision model to be assigned as "homework" to specific experts. Moreover, the fact that the product engineers not only understood but even helped to build the formal model is also important for its maintenance, for which the engineers themselves will mainly be responsible.

DMN achieves its readability by offering a visual notation (the Decision Requirements Diagram or $D R D$ ) to decompose a big decision into smaller subdecisions, a visual decision table notation to model individual decisions, and the intuitive $S$-FEEL language that can be used inside the decision tables.

However, the simplicity of DMN comes at a cost. While we found that this format elegantly handled the majority of the decision processes of our case study, a limited number of key decisions simply did not fit into the framework. In this paper, we propose an extension to the DMN standard, which aims to make it better suited for complex real-life situations. In [3], it was shows that DMN can be seen as an intuitive notation for certain formulas in First-Order Logic (FO). Following this approach, we will define the newly introduced constraint tables by means of a transformation to FO. The main advantage of this approach is that we can then feed this representation into a model generation system for FO, such as IDP [4], MiniSat [5] or z3 [6], and thereby immediately obtain an implementation of our extension.

In Sec. 2, we describe the business case that we will use as running example. We explain the difficulties we faced when formalizing this case in DMN (Sec. 3), followed by proposing constraint tables as an extension to DMN in Sec. 4 Then, we discuss the semantics of DMN decision tables and the newly introduced constraint tables (Sec. 5). Section 6 discusses how constraint tables facilitates handling the running example. Related work is presented in the Sec.7. Finally, conclusions and future work are discussed in Sec. 8 .

\section{Running example : Product Design}

We will apply DMN and our extension to a real-life use case at a company that manufactures highly specialized products to order. This use case is discussed extensively in [2]. In this section, we recall a simplified version.

The task of the company's product engineers is to design a product that consists of two mandatory components: a body and a spring. In addition, there may be a third component, called a wiper. The engineers are given a number of specifications: the desired dimension of the product, the temperature range and pressure in which it should function.

They need to determine: 1. Which type of body (closed or open) to use; 2. What material to use for it; 3 . Whether to use a normal or a thick spring.

They have to make these choices in such a way that: 1 . The materials used can cope with the given temperature range; 2 . When the materials shrink due to cold, the spring should prevent the component from falling out of the cavity in which it is placed; 3. A wiper is included if the component is to operate in a dirty environment. The materials have different costs, with cheaper materials 
typically being weaker than more expensive ones. In general, the engineers look for the cheapest design that will work.

\section{DMN Case}

We present a DMN model that is based on the typical decision procedure followed by the engineers. As we will show, parts of this procedure fit naturally into DMN, while others require cumbersome work-arounds. Fig. 1 shows our DRD, which captures the general structure of the decision logic. It starts from customer requirements at the bottom and has the decisions that must be made at the top, with certain subdecisions in between. Each rectangle corresponds to a decision table, which can be found in Fig,2. The different components of such a table are identified in Fig, 3. The (dark) green headers represent input columns, while the (light) blue headers are output columns. As can be seen in these figures, all of our tables use the $U$ (nique) hit policy, which means that the conditions on the input columns in different rows must be mutually exclusive.

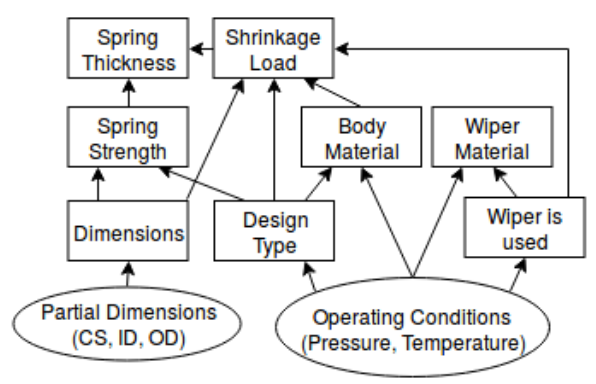

Fig. 1: DRD model of application.

To design a component, the engineers typically proceed as follows:

1. There are 3 relevant dimensions: the outer diameter (OD) of the component, its inner diameter (ID) and its cross-section (CS). The customer provides 2 of these and the engineer computes the third $(C S=$ $(O D-I D) / 2)$. The corresponding DMN table consists of three different rows (one per dimension that might be missing), that all contain essen-

tially the same information.

2. An initial design type is chosen, depending on the required operating conditions. This decision fits well within the mold of a DMN table.

3. Based on the temperature restrictions and design type, the "best" material is selected for the body. When representing this decision in a DMN table, we lose the distinction between physical constraints (i.e., the fact that some materials simply cannot cope with certain temperatures) and preferences (i.e., the fact that some material is not chosen because a cheaper material is available). Because this distinction is lost, is not clear how to update this table when, e.g., the price of a certain material drops: there may be cases in which this material was originally not chosen because it was too expensive, but we cannot discern them from cases where the material was not chosen because it would melt.

4. The expected shrinkage is computed, based on the design type, dimensions, temperature and selected materials. While we omit the details, the formula for this calculation can easily be placed in the "Shrinkage Load" table.

5. Based on the selected design type, the spring strength of a standard spring is computed. If this is enough to cope with the expected shrinkage, the standard 
spring is selected. Otherwise, the engineers switch to a thick spring and recompute the spring strength of the design. Since DMN does not allow to "recompute" a value, the "Spring Strength" table computes both the spring strength that the design would have with a standard spring and that with a thick spring. The "Spring Thickness" table then uses these two values to decide which spring type to use. Because of this, the DRD has an edge from "Spring Strength" to "Spring Thickness". This is counterintuitive, because in reality it is the thickness of the spring that determines the design's spring strength, not the other way around.

6. If, for an open design, even the thick spring does not provide enough spring strength, the engineers' final option is to switch to a closed design type, but since this is an initial choice upon which all further choices are based, this means that the engineers essentially start over the entire process from scratch. If the thick spring's strength does not suffice, our DMN table will fail to assign a value to "Spring Thickness" (i.e., it will be null). This will alert the user that something is wrong, but the backtracking step of redoing the entire design process with a closed design is not possible in DMN. For this reason, we have implemented a workaround in which the "Design Type" table is overly cautious: in low temperatures, it will always choose a closed design, even in cases when an open design could suffice. This is sub-optimal, but avoids the possibility of failing the spring thickness check at the end.

\begin{tabular}{|l|l|l|l|l|l|l|}
\hline \multicolumn{3}{|l|}{ Dimensions } \\
\cline { 1 - 4 } U & OD & ID & CS & OD & ID & CS \\
\hline 1 & null & - & - & ID $+2^{*} \mathrm{CS}$ & & \\
\hline 2 & - & null & - & & OD $-2^{*} \mathrm{CS}$ & \\
\hline 3 & - & - & null & & & $(\mathrm{OD}-\mathrm{ID}) / 2$ \\
\hline
\end{tabular}

\begin{tabular}{|l|l|l|l|}
\hline \multicolumn{3}{|l|}{ Body Material } & \multicolumn{2}{l}{} \\
\cline { 1 - 2 } & $\begin{array}{l}\text { Tempe- } \\
\text { rature }\end{array}$ & $\begin{array}{l}\text { Design } \\
\text { Type }\end{array}$ & $\begin{array}{l}\text { Body } \\
\text { Material }\end{array}$ \\
\hline$\ldots$ & $\ldots$ & $\ldots$ & $\ldots$ \\
\hline 5 & {$[-50,80]$} & Closed & M1 \\
\hline 7 & $(80,150]$ & - & M3 \\
\hline$\ldots$ & $\ldots$ & $\ldots$ & $\ldots$ \\
\hline
\end{tabular}

\begin{tabular}{|l|l|l|}
\hline \multicolumn{2}{|l|}{ Spring Thickness } \\
\hline U & Shrinkage Load & Spring Thickness \\
\hline & & \\
1 & $<=$ Standard Spring Strength & Standard \\
\hline 2 & (Standard Spring Strength,Thick Spring Strength] & Thick \\
\hline
\end{tabular}

\begin{tabular}{|c|c|c|c|}
\hline \multicolumn{2}{|c|}{ Spring Strength } & \multirow[b]{2}{*}{$\begin{array}{l}\text { Standard } \\
\text { Spring } \\
\text { Strength }\end{array}$} & \multirow[b]{2}{*}{$\begin{array}{l}\text { Thick } \\
\text { Spring } \\
\text { Strength }\end{array}$} \\
\hline U & $\begin{array}{l}\text { Design } \\
\text { Type }\end{array}$ & & \\
\hline 1 & Closed & 15 & 20 \\
\hline 2 & Open & 7 & 11 \\
\hline
\end{tabular}

\begin{tabular}{|c|l|l|l|l|l|}
\hline \multicolumn{2}{|l}{ Shrinkage Load } & $(100,150]$ & $>-50$ & Open \\
\cline { 1 - 3 } & $\begin{array}{l}\text { Design } \\
\text { Type }\end{array}$ & Shrinkage Load \\
\hline 1 & $\ldots$ & $\mathrm{f}($ Cross Section, Material of Body, Temperature $)$ \\
\hline
\end{tabular}

Fig. 2: Decision tables for component design

\begin{tabular}{|c|c|c|c|}
\hline Table Name & $\rightarrow$ Wiper is used & & Input expression \\
\hline Hit policy & \begin{tabular}{l|l}
$U_{X} C$ & Dirty Environment
\end{tabular} & Wiper is used & Output expression \\
\hline Completeness indicator & Yes/No & Yes/No & \\
\hline Facet & Yes & Yes & Output entries \\
\hline Rule number & No & No & Input entries \\
\hline
\end{tabular}

Fig. 3: The constitutive elements of the "Wiper is used" decision table 


\section{Constraint Tables}

In this section, we extend DMN with constraint tables. This will allow a more direct representation of the constraints that are relevant for constructing a design and will thereby avoid the issues highlighted in the previous section.

A DMN decision table uniquely defines the value of its outputs. This is due to two properties. First, while cells in an input column may contain different kinds of S-FEEL expression, only single values can be used in output columns. Therefore, if a row matches the input, the corresponding single value must be assigned to the output. Second, DMN allows to designate a default value for each output column: if no row matches the input, then the output takes on this default value. Alternatively, when there is no default, the output is assigned the special value $n u l l$, which is typically taken to indicate an error in the specification.

Our new constraint tables change both of these properties. First, we allow the same S-FEEL expressions that can be used in input columns to appear in output columns as well. For instance, the Material of Body table in Fig. 4 states that if a closed design is used, material M2 cannot be used, without specifying which of the other materials should be used in its place. Second, the rows of a constraint table are viewed as logical implications, in the sense that if the conditions on the inputs are satisfied, then the conditions on the outputs must also be satisfied. This means that if, for instance, none of the rows are applicable, the outputs can take on an arbitrary value, as opposed to being forced to null (in constraint tables, no default values can be assigned).

We introduce a new hit policy to identify constraint tables. We call this the Every hit policy, denoted as $E^{*}$, because it expresses that every one of these implications must be satisfied. Consider, for intance, the Design Type table in Fig. 4. Regardless of hit policy, this table states that if the pressure exceeds 150 , the design type must be open. The effect of its $E^{*}$ hit policy is seen when the pressure does not exceed 150. In this case, our constraint table imposes no restriction on the design: an open and a closed design are both possible. The Material of Body table combines the $E *$ hit policy with the ability to use SFEEL expressions in the output. It states that if the design is closed, material $M 2$ cannot be used for the body. Again, if an open design is used, no constraints are imposed on the body material.

\section{The semantics of DMN and constraint tables}

Calvanese et al. express the formal semantics of decision tables in First-Order Logic 3. We will use this as a starting point for the semantics of constraint tables, so we repeat some of this formalization here. For reasons of space, we restrict attention to the fragment of DMN used in our running example.

Each column in a decision table corresponds to an FO constant, that is mentioned in the column's header. Each cell in the column represents a condition that this constant may satisfy. Such a condition $Q$ is transformed into an FO formula $\Phi_{Q}(x)$ in one free variable $x$. The easiest case is when the condition 
consists of a term $T$ (a term is either a constant of an $n$-ary function applied to $n$ terms), which is short for the equality " $=T "$ ". In this case, $\Phi_{Q}(x)$ is the formula $x=T$. If $Q$ is an interval $[i, j]$, then $\Phi_{Q}(x)$ is $x \geq i \wedge x \leq j$. If $Q$ is a list $\left(C_{1}, \ldots, C_{n}\right)$ of constants, then $\Phi_{Q}$ is $x=C_{1} \vee \ldots \vee x=C_{n}$. Other kinds of intervals and comparisons are defined in a similar way. Table 1 summarizes a number of the possibilities.

\begin{tabular}{|c||c|c|c|c|c|c|}
\hline$Q$ & - & $T$ & $\operatorname{not}(T)$ & $\leq T$ & $(i, j]$ & $Q_{1}, Q_{2}$ \\
\hline$\Phi_{Q}(x)$ & true & $x=T$ & $\neg \Phi_{T}$ & $x \leq T$ & $x>i \wedge x \leq j$ & $x=\Phi_{Q_{1}} \vee x=\Phi_{Q_{2}}$ \\
\hline
\end{tabular}

Table 1: The FO translation $\Phi_{Q}$ of different S-FEEL conditions $Q$.

A row in a decision table corresponds to the conjunction of all these condition formulas, applied to their respective column headers. For instance, the second row of the "Design Type" table corresponds to:

$$
\text { Pressure }>100 \wedge \text { Pressure } \leq 150 \wedge \text { Temp } \geq-50 \wedge \text { DesignType }=\text { Closed } .
$$

An entire table then corresponds to the disjunction of all its rows, e.g.:

$$
(\text { Pressure }>100 \wedge \text { Pressure } \leq 150 \wedge \text { Temp } \geq-50 \wedge \text { DesignType }=\text { Closed })
$$$$
\vee(\text { Pressure } \leq 100 \wedge \text { true } \wedge \text { DesignType }=\text { Closed }) \vee \ldots
$$

In constraint tables, we represent each condition $Q$ in a table row by the same formula $\Phi_{Q}$, as shown in Table 1 . The difference with decision tables lies in how we combine these individual formulas $\Phi_{Q}$. In a constraint table, a row no longer corresponds to a conjunction, but to an implication. To be more concrete, if the first $m$ columns of the table are inputs and the next $n-m$ columns are output, a row $r=\left(Q_{1}, \ldots, Q_{m}, Q_{m+1}, Q_{n}\right)$ corresponds to the implicative formula $\Phi_{\vec{H}}^{\overrightarrow{ }}(r)$ :

$$
\bigwedge_{i=0}^{m} \Phi_{Q_{i}} \Rightarrow \bigwedge_{i=0}^{n} \Phi_{Q_{m+i}} .
$$

For instance, the first row of the "Material of Body" table corresponds to the formulas DesignType $=$ Closed $\Rightarrow$ MaterialOfBody $\neq M 2$. The semantics of constraint table $T$ is then defined as the conjunction $\bigwedge_{r \in R} \Phi_{\vec{H}}^{\overrightarrow{ }}(r)$.

\section{Discussion}

We discuss some features of our constraint table representation of the running example in more detail.

Dimensions DMN tools use decision tables in a strictly feed-forward way, deriving outputs from inputs. By contrast, when we feed the constraint tables into a FO model generation tool, this can use the constraint in different ways. For instance, it can use the Dimensions table to derive eithe CS, OD or ID from the other two.

Design Type The only physical design type constraint is that a closed design is not possible for pressures larger than 150. Nevertheless, the corresponding 


\begin{tabular}{|c|c|c|c|c|c|c|c|c|c|c|}
\hline \multicolumn{3}{|c|}{ Shrinkage Check } & \multicolumn{2}{|c|}{ Design Type } & & \multicolumn{3}{|c|}{ Shrinkage Load } & & \\
\hline$E^{*}$ & \multicolumn{2}{|c|}{ Shrinkage Load } & $E^{*}$ & Pressure & \begin{tabular}{|l|} 
Design \\
Type \\
\end{tabular} & $\mathrm{U}$ & $\begin{array}{l}\text { Design } \\
\text { Type }\end{array}$ & \multicolumn{3}{|c|}{ Shrinkage Load } \\
\hline 1 & \multicolumn{2}{|c|}{$<$ Spring Strength } & 1 & $>150$ & Open & 1 & $\ldots$ & \multicolumn{3}{|c|}{$\mathrm{f}(\mathrm{CS}$, Material of Body, Temperature $)$} \\
\hline \multicolumn{4}{|c|}{ Spring Strenght } & & \multicolumn{3}{|c|}{ Dimensions } & \multicolumn{2}{|c|}{ Operating Conditions } & \\
\hline & \multirow{2}{*}{$\begin{array}{l}\text { Design } \\
\text { Type }\end{array}$} & \multirow{2}{*}{\multicolumn{2}{|c|}{$\begin{array}{l}\text { Spring } \\
\text { Thickness }\end{array}$}} & \multirow{2}{*}{\begin{tabular}{|l} 
Spring \\
Strength
\end{tabular}} & $\mathrm{U}$ & \multicolumn{2}{|l|}{ CS } & \multirow{2}{*}{\begin{tabular}{|l|}
$E^{*}$ \\
1
\end{tabular}} & $\begin{array}{l}\text { Material of } \\
\text { body }\end{array}$ & Temp \\
\hline $\mathrm{U}$ & & & & & 1 & \multicolumn{2}{|c|}{$(\mathrm{OD}-\mathrm{ID}) / 2$} & & M1 & {$[-100,150]$} \\
\hline 1 & Closed & \multicolumn{2}{|c|}{ Normal } & 15 & & \multirow{2}{*}{\multicolumn{2}{|c|}{ Material of Body }} & \multirow{2}{*}{\begin{tabular}{|r|}
2 \\
$\ldots$ \\
\end{tabular}} & M2 & {$[-120,80]$} \\
\hline 2 & Closed & \multicolumn{2}{|c|}{ Thick } & 20 & Mat & & & & $\ldots$ & $\ldots$ \\
\hline 3 & Open & \multicolumn{2}{|c|}{ Normal } & 7 & $E^{*}$ & \multicolumn{2}{|c|}{ Design Type } & \multicolumn{2}{|c|}{ Material of Body } & \\
\hline 4 & Open & Thicl & & 11 & 1 & \multicolumn{2}{|c|}{ Closed } & \multicolumn{2}{|l|}{ Not(M2) } & \\
\hline
\end{tabular}

Fig. 4: Combination of constraint and decision tables

table in our original DMN representation (Fig. 3) also contained the temperature as an input column. This was to avoid running into cases in which the shrinkage cannot be addressed by simply adding a thick spring to an open design. By contrast, our constraint representation states directly that a design type must be chosen in accordance with the constraints expressed in the Design Type table and the Shrinkage Load table, avoiding the need for the original work-around.

Materials Each material has a minimum/maximum temperature, defined in the Operating Conditions table. It states that, if a material is used, the operating temperature should be within the allowed temperature range of that material.

\section{Related Work}

The limitations of DMN decision tables have been recognized before. In addition to the S-FEEL language that we have used in this paper, the DMN standard itself also puts forward the more general FEEL language to allow much more complex expressions. However, FEEL is a full programming language with its own syntax, and hence not really suitable for direct use by domain experts 1 .

Also a number of DMN tools provide ways to deal with the limitations. For instance, OpenRules allows to insert Java-snippets to express complex parts of the logic. The advantage of this approach is that it still keeps the overall idea of decision tables that can be maintained by business experts, while allowing an IT-expert to code specific complex parts of the decision logic. In addition to allowing imperative code snippets, several approaches also allow DMN to be extended by more declarative representations. For instance, the aforementioned OpenRules also offers an interface to a constraint solver [7], while [8] allows DMN to be enriched with domain knowledge expressed in Description Logic.

These papers share with ours the goal of extending DMN to be able to cope with the complex knowledge that typically arises in real-world problems. However, they extend DMN's decision tables with a completely separate formalism, that is intended to be used by knowledge engineers rather than DMN approach. By contrast, our goal in this paper is to investigate whether it is possible to allow the more complex knowledge to also be expressed within DMN's table format, allowing it to be maintained by domain experts as well. 


\section{Conclusions and future work}

The DMN standard is currently gaining traction in industry. It offers an easyto-use decision table format for representing decision logic, which allows domain experts to write and maintain the models, without requiring interventions by IT staff. Despite DMN's increasing popularity, it often still lacks the expressivity necessary to tackle real-world problems. In this paper, we have given an example of such a real-world problem, we have presented a DMN model for it, and discussed why this model is not really satisfactory.

Our main contribution is the extension of DMN with constraint tables. Crucially, constraint tables allow complex knowledge to be expressed, while still retaining the appealing visual and "syntax-less" representation that has made DMN itself a success. While extending DMN in this way may make the language harder to learn, we believe that domain experts will still be able to cope with constraint tables in DMN. While perhaps building a model from scratch would be more of a challenge, we are confident that, at least, if a model has initially been built in collaboration with a knowledge engineer, the domain experts will be able to maintain it without further help. Indeed, when looking at the constraint representation of our running example, we believe that the meaning of each of these tables will be clear enough to domain experts to make this feasible.

Following [3], we have defined the semantics of constraint tables by a transformation to first-order logic. The resulting formulas can be fed into a solver, providing a working implementation. Currently, we still perform this transformation by hand, but we are working on a fully automatic implementation.

\section{References}

1. OMG: Decision Model and Notation 1.2 (2018)

2. Aerts, B., Vennekens, J.: Application of Logic-Based Methods to Machine Component Design. In: Technical Communications of the 34th International Conference on Logic Programming (ICLP 2018). (2018)

3. Calvanese, D., Dumas, M., Laurson, Ü., Maggi, F.M., Montali, M., Teinemaa, I.: Semantics and analysis of DMN decision tables. In: International Conference on Business Process Management, Springer (2016) 217-233

4. De Cat, B., Bogaerts, B., Bruynooghe, M., Janssens, G., Denecker, M. In: Predicate logic as a modeling language: the IDP system. (2018) 279-323

5. Eén, N., Sörensson, N.: An extensible sat-solver. In: International conference on theory and applications of satisfiability testing, Springer (2003) 502-518

6. De Moura, L., Bjørner, N.: Z3: An efficient smt solver. In: I'al conference on Tools and Algorithms for the Construction and Analysis of Systems, Springer (2008)

7. Feldman, J.: Representing and solving rule-based decision models with constraint solvers. Volume 7018. (01 2011) 208-221

8. Calvanese, D., Dumas, M., Maggi, F.M., Montali, M.: Semantic DMN: Formalizing decision models with domain knowledge. In: International Joint Conference on Rules and Reasoning, Springer (2017) 